converges better the shorter the cylinder, so that by one of these formulas the calculation may always be made. Coffin has also given a formula by elliptic integrals, which is convenient when tables are at hand. Mr. Gordon Fulcher has

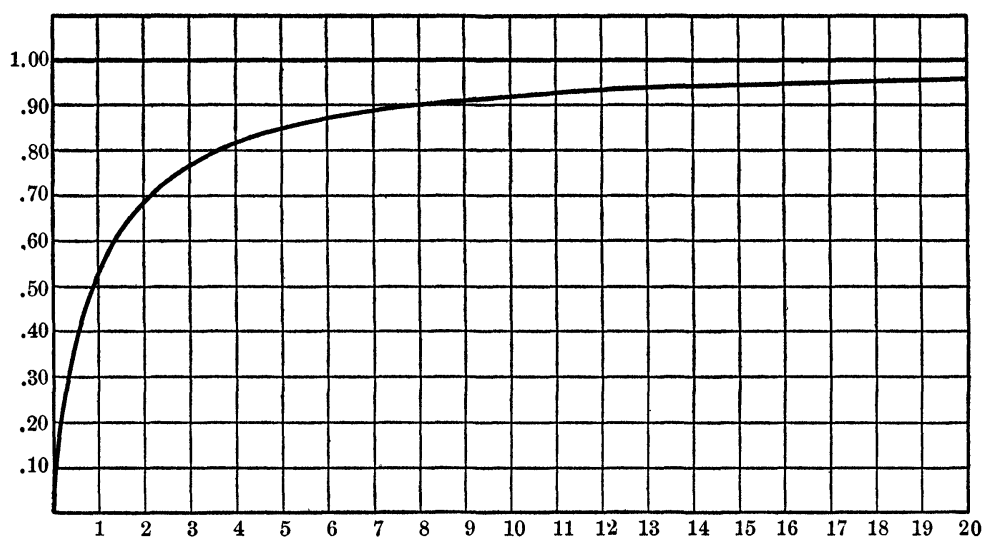

calculated by the three methods values for some twenty ratios, from which he has constructed the annexed graph of $L / 4 \pi^{2} a^{3} m^{2} z^{2}$, that is the factor of correction for the ends of the solenoid. When the length is ten times the diameter, five terms of (20) give nine figures of the result.

Clark University, July, 1907.

\title{
ON THE APSIDAL ANGLE IN CENTRAL ORBITS.
}

BY DR. F. L. GRIFFIN.

(Read in part before the American Mathematical Society, April 27, 1907.)

There are two well known laws of central force all of whose trajectories have the same apsidal angle, whatever be the apsidal values of the radius vector, viz., that of Newton and the law that the force varies directly as the distance. For both of these laws the orbits are all conic sections, the apsidal angle being $\pi$ in the former case, and $\frac{1}{2} \pi$ in the latter. Generally, however, the apsidal angle depends upon the apsidal values of the radius vector.

In this paper are considered only those laws of central force for which the force is a function of the distance, having a finite 
derivative except at a finite number of distances in any interval. For all such laws, and for all orbits lying wholly within any interval throughout which this derivative is finite, four theorems will be proved. If, for a given law, the derivative is finite for all values of the distance, the theorems hold for all the trajectories. The criteria obtained find immediately two applications : (1) To orbits in the equatorial planes of attracting spheroids - incidentally explaining a well known phenomenon in the motion of the fifth satellite of Jupiter; and (2) to the trajectories for various simple laws of force.

Let $u$ denote the reciprocal of the radius vector, and $u^{2} P(u)$, the force ; and let $P^{\prime}(u)=d[P(u)] / d u$. Evidently, the derivative of the force with respect to the distance is finite if, and only if, $P^{\prime}(u)$ is finite.

Theorem I. In order that the apsidal angle be greater than $\pi$ (equal to $\pi$, or less than $\pi$ ) in every trajectory for a given law, it is necessary and sufficient that $P^{\prime}(u)$ be (respectively) everywhere positive (zero, or negative).

The proof is got by considering the definite integral which gives the apsidal angle. If $h$ be the constant of areas and $\theta$ the longitude, the differential equation of any orbit under the given law is

$$
h^{2} u^{2}\left(u+d^{2} u / d \theta^{2}\right)=u^{2} P(u) \equiv \frac{1}{2} u^{2} F^{\prime}(u) .
$$

$[P(u)$, since it has a derivative, is continuous and therefore integrable.] After integration, one has

$$
h^{2}\left[u^{2}+(d u / d \theta)^{2}\right]=c+F(u),
$$

$c$ being a constant. Or, if $\alpha$ and $\beta[\beta>\alpha]$ be the apsidal values of $u$,

$$
\begin{aligned}
\left(\frac{d u}{d \theta}\right)^{2} & =\left(\beta^{2}-u^{2}\right)+\frac{1}{h^{2}}[F(u)-F(\beta)] \\
& =\left(\alpha^{2}-u^{2}\right)+\frac{1}{h^{2}}[F(u)-F(\alpha)] .
\end{aligned}
$$

Hence the apsidal angle is given by

$$
\Theta=\int_{a}^{\beta} \frac{d u}{\sqrt{\beta^{2}-u^{2}+\frac{1}{h^{2}}[F(u)-F(\beta)]}} .
$$


Sufficiency of the condition on $P^{\prime}(u)$. Compare $\Theta$ with $\pi$, the apsidal angle in the case of Newton's law, where $P(u)=N$, a constant. For the latter law the second member of (1) is $(\beta-u)\left[\beta+u-2 N / h^{2}\right]$. Hence, if $N$ be selected,

$$
N=\frac{1}{2} h^{2}(\alpha+\beta),
$$

the Keplerian ellipse will have the same constant of areas and the same apsidal values of $u$ as the original orbit. Therefore

$$
\begin{aligned}
\pi & =\int_{a}^{\beta} \frac{d u}{\sqrt{\beta^{2}-u^{2}+\frac{2 N}{h^{2}}(u-\beta)}}, \\
\Theta-\pi & =\int_{a}^{\beta}\left\{\frac{1}{\sqrt{\beta^{2}-u^{2}+\frac{1}{h^{2}}[F(u)-F(\beta)]}}\right. \\
& \left.-\frac{1}{\sqrt{\beta^{2}-u^{2}+\frac{2 N}{h^{2}}(u-\beta)}}\right\} d u .
\end{aligned}
$$

Consider now the function

$h^{2}\left\{\beta^{2}-u^{2}+\frac{2 N}{h^{2}}(u-\beta)\right\}-h^{2}\left\{\beta^{2}-u^{2}+\frac{1}{h^{2}}[F(u)-F(\beta)]\right\}$, or

$$
\Phi(u) \equiv 2 N(u-\beta)-F(u)+F(\beta) .
$$

Since in both orbits $d u / d \theta$ vanishes at $u=\alpha$, and at $u=\beta$, it follows that

$$
\Phi(\alpha)=\Phi(\beta)=0
$$

Also,

$$
\Phi^{\prime \prime}(u)=-F^{\prime \prime}(u)=-2 P^{\prime}(u) .
$$

Suppose now that $P^{\prime}(u)>0$ for $\alpha \leqq u \leqq \beta$. Then $\Phi^{\prime \prime}(u)<0$, and consequently $\Phi^{\prime}(u)$ decreases throughout the interval $(\alpha, \beta)$. Now since $\Phi^{\prime}(u)$ has a derivative it is continuous, and therefore vanishes at most once for $\alpha \leqq u \leqq \beta$. But, from (6), $\Phi^{\prime}(u)$ must vanish once, by Rolle's theorem. Since $\Phi^{\prime}(u)$ decreases from $u=\alpha$ to $u=\beta$ and vanishes between $\alpha$ and 
$\beta, \Phi^{\prime}(\alpha)>0$ and $\Phi^{\prime}(\beta)<0$. Consequently $\Phi(u)>0$ for $\alpha<u<\beta$.

This shows that the integrand (4) is everywhere positive, whence $\Theta-\pi>0$ or $\Theta>\pi$.

Similarly, suppose $P^{\prime}(u)$ everywhere negative ; then $\Phi(u)<0$, the integrand is everywhere negative, and $\Theta<\pi$. Likewise for $P^{\prime}(u)=0$, the integrand is zero throughout, and $\Theta=\pi$. Thus, the sufficiency of the condition is established.

Necessity of the condition on $P^{\prime}(u)$. Suppose $P^{\prime}(u)$ changes sign within the interval $(\alpha, \beta)$. Then within some interval $\left(\alpha_{1}, \beta_{1}\right), P^{\prime}(u)>0$; and within some interval $\left(\alpha_{2}, \beta_{2}\right), P^{\prime}(u)<0$. Hence, by the sufficiency proof, all orbits lying wholly within $\left(\alpha_{1}, \beta_{1}\right)$ have their apsidal angles greater than $\pi$; and all those lying within $\left(\alpha_{2}, \beta_{2}\right)$ have their apsidal angles less than $\pi$. But this does not fulfill the requirement that $\Theta$ be greater than $\pi$ (equal to $\pi$, or less than $\pi$ ) for all orbits lying within $(\alpha, \beta)$. Hence the condition is necessary.

THEOREM II. If $P^{\prime}(u)$ is positive, and increases with $u$ or is constant, then, in those families of orbits one of whose apsidal distances is constant, the apsidal angle increases as the second apsidal distance decreases.

[For orbits having a common pericentral distance this means that the apsidal angle is greater the more nearly circular the orbit; for orbits having a common apocentral distance the reverse is true.]

Case I. Let $1 / \beta$ be the common pericentral distance of two orbits, whose apocentral distances are $1 / \alpha_{1}$ and $1 / \alpha_{2}$, and whose constants of areas are $h_{1}$ and $h_{2}, h_{2}>h_{1}$. Now, from the differential equations (1) of the two orbits, it follows that for equal values of $u_{1}$ and $u_{2}$

$$
h_{2}^{2}\left(\frac{d u}{d \theta}\right)_{2}^{2}=h_{1}^{2}\left(\frac{d u}{d \theta}\right)_{1}^{2}+\left(h_{2}^{2}-h_{1}^{2}\right)\left(\beta^{2}-u^{2}\right) .
$$

And, since $(d u / d \theta)_{1}$ is real for $\beta \geqq u_{1} \geqq \alpha_{1}$, the same is true of $(d u / d \theta)_{2}$, and moreover $(d u / d \theta)_{2}^{2}>0$ at $u_{2}=\alpha_{1}$. Hence the interval $\left(\alpha_{1}, \beta\right)$ is one of real motion for orbit 2 ; and $\alpha_{2}$ lies outside the interval, so that $\alpha_{2}<\alpha_{1}$. The apsidal angles $\Theta_{1}$ and $\Theta_{2}$ are given by

$$
\Theta_{i}=\int_{a_{i}}^{\beta} \frac{d u_{i}}{\sqrt{\beta^{2}-u_{i}^{2}+\frac{1}{h_{i}^{2}}\left[F\left(u_{i}\right)-F(\beta)\right]}} \quad(i=1,2) .
$$


To compare these two angles, make the following linear substitutions: $u_{1}=a \beta+b u, u_{2}=u$, where

$$
a=\frac{\alpha_{1}-\alpha_{2}}{\beta-\alpha_{2}}, \quad b=\frac{\beta-\alpha_{1}}{\beta-\alpha_{2}}=1-a .
$$

Both $a$ and $b$ are evidently positive and less than unity; and $u_{1}>u$ if $\beta>u$. Then $\Theta_{1}-\Theta_{2}$ becomes

$$
\begin{array}{r}
\Theta_{1}-\Theta_{2}=\int_{\alpha_{2}}^{\beta}\left\{\frac{b}{\sqrt{\beta^{2}-(a \beta+b u)^{2}+\frac{1}{h_{1}^{2}}[F(a \beta+b u)-F(\beta)]}}\right. \\
\left.-\frac{1}{\sqrt{\beta^{2}-u^{2}+\frac{1}{h_{2}^{2}}[F(u)-F(\beta)}}\right\} d u,
\end{array}
$$

for $u=\alpha_{2}$ at $u_{1}=\alpha_{1}$. A function similar to that used in proving Theorem I will be defined here,

$$
\begin{aligned}
\Psi(u) & \equiv h_{1}^{2} b^{2}\left[h_{2}^{2}\left(\beta^{2}-u^{2}\right)+F(u)-F(\beta)\right] \\
& -h_{2}^{2}\left[h_{1}^{2}\left(\beta^{2}-(a \beta+b u)^{2}\right)+F(a \beta+b u)-F(\beta)\right] .
\end{aligned}
$$

Since in both orbits $d u / d \theta$ vanishes for $u=\alpha_{2}$ and for $u=\beta$,

$$
\Psi\left(\alpha_{2}\right)=\Psi(\beta)=0 .
$$

Evidently

or

$$
\begin{aligned}
b^{-2} \Psi^{\prime \prime}(u) & =h_{1}^{2} F^{\prime \prime}(u)-h_{2}^{2} F^{\prime \prime}(a \beta+b u) \\
& =2 h_{1}^{2} P^{\prime}(u)-2 h_{2}^{2} P^{\prime}(a \beta+b u)
\end{aligned}
$$

$$
\frac{1}{2} b^{-2} \Psi^{\prime \prime}(u)=\left(h_{1}^{2}-h_{2}^{2}\right) P^{\prime}(u)-h_{2}^{2}\left[P^{\prime}\left(u_{1}\right)-P^{\prime}(u)\right] .
$$

Since $h_{2}>h_{1}$ and $P^{\prime}(u)$ is positive, the first term on the right side of (12) is negative, and since $P^{\prime}(u)$ increases with $u$ or is constant, and $u_{1}>u$ for $\beta>u$, the second term is negative or zero. Hence $\Psi^{\prime \prime}(u)<0$ for $u<\beta$, and $\Psi^{\prime}(u)$ decreases from $u=\alpha_{2}$ to $u=\beta$, vanishing at most once, because continuous. But, from (11), it vanishes once; this shows that $\Psi^{\prime}\left(\alpha_{2}\right)>0$ and $\Psi^{\prime}(\beta)<0$. Consequently $\Psi(u)>0$ for $\beta>u>\alpha_{2}$, and the integrand is positive throughout, showing that $\Theta_{1}-\Theta_{2}>0$. 
Now it was shown that $h_{2}>h_{1}$ implies $\alpha_{2}<\alpha_{1}$; also it is obvious that $h_{2}=h_{1}$ implies $\alpha_{2}=\alpha_{1}$. It follows, therefore, that the greater the value of $\alpha$-that is, the more nearly circular the orbit - the greater is the apsidal angle. The theorem is thus established for families of orbits with the same pericentral distance; it remains to examine those having the apocentral distance constant.

Case II. Let $1 / \alpha$ be the common apocentral distance of two orbits whose pericentral distances are $1 / \beta_{1}$ and $1 / \beta_{2}$, and whose constants of areas are $h_{1}$ and $h_{2}, h_{2}>h_{1}$. An examination of the differential equations corresponding to (1) shows that for equal values of $u_{1}$ and $u_{2}$

$$
h_{1}^{2}\left(\frac{d u}{d \theta}\right)_{1}^{2}=h_{2}^{2}\left(\frac{d u}{d \theta}\right)_{2}^{2}+\left(h_{1}^{2}-h_{2}^{2}\right)\left(\alpha^{2}-u^{2}\right) .
$$

And, since $(d u / d \theta)_{2}$ is real for $\beta_{2} \geqq u_{2} \geqq \alpha$, the same is true of $(d u / d \theta)_{1}$, and moreover $(d u / d \theta)_{1}^{2}>0$ at $u_{1}=\beta_{2}$. Hence the orbit 1 is real throughout the interval $\left(\alpha, \beta_{2}\right)$, and beyond. Therefore $\beta_{1}>\beta_{2}$. The difference of the apsidal angles may then be written

$$
\begin{gathered}
\Theta_{1}-\Theta_{2}=\int_{a}^{\beta_{1}}\left\{\frac{1}{\sqrt{\alpha^{2}-u^{2}+\frac{1}{h_{1}^{2}}[F(u)-F(\alpha)]}}\right. \\
\left.-\frac{f}{\sqrt{\alpha^{2}-(e \alpha+f u)^{2}+\frac{1}{h_{2}^{2}}[F(e \alpha+f u)-F(\alpha)]}}\right\} d u,
\end{gathered}
$$

where

$$
e=\frac{\beta_{1}-\beta_{2}}{\beta_{1}-\alpha}, f=\frac{\beta_{2}-\alpha}{\beta_{1}-\alpha}=1-e .
$$

Evidently both $e$ and $f$ are positive and less than unity; and $e \alpha+f u<u$ for $\alpha<u$. Let

$$
\begin{array}{r}
\chi(u) \equiv h_{1}^{2}\left[h_{2}^{2}\left(\alpha^{2}-(e \alpha+f u)^{2}\right)+F(e \alpha+f u)-F(\alpha)\right] \\
-f^{2} h_{2}^{2}\left[h_{1}^{2}\left(\alpha^{2}-u^{2}\right)+F(u)-F(\alpha)\right] .
\end{array}
$$

Then, for the same reason as in (11),

and

$$
\chi(\alpha)=\chi\left(\beta_{1}\right)=0 ;
$$




$$
\frac{1}{2 f^{2}} \chi^{\prime \prime}(u)=\left(h_{1}^{2}-h_{2}^{2}\right) P^{\prime}(u)-h_{1}^{2}\left[P^{\prime}(u)-P^{\prime}(e \alpha+f u)\right] .
$$

Since both terms of the right member of (17) are negative for $u>\alpha, \chi^{\prime \prime}(u)<0$ so that the discussion of $\Psi(u)$ in Case I applies equally to $\chi(u)$ here. Hence, $\Theta_{1}-\Theta_{2}>0$, or $\Theta_{1}>\Theta_{2}$. Finally, then, it follows that the smaller the pericentral distance - that is, the less nearly circular the orbit - the greater the apsidal angle.

Corollary. If two orbits have no apsidal distance in common, both apsidal distances in the first being less than the corresponding distances in the second, the apsidal angle in the first is greater than that in the second.

For, let the apocentral and pericentral distances in orbit 1 be, respectively, $1 / \alpha_{1}$ and $1 / \beta_{1}$, and in orbit $2,1 / \alpha_{2}$ and $1 / \beta_{2}$. Consider a new orbit - orbit 3 - with pericentral distance $1 / \beta_{1}$ and apocentral distance $1 / \alpha_{2}$. Then, by Case $I, \Theta_{1}>\Theta_{3}$; and by Case II, $\Theta_{3}>\Theta_{2}$. Hence, $\Theta_{1}>\Theta_{2}$.

Theorem III. If $P^{\prime}(u)$ is negative, and a decreasing function of $u$ or constant, then in those families of orbits one of whose apsidal distances is constant, the apsidal angle increases with the other apsidal distance.

The proof is got as in Theorem II, except that $\Psi^{\prime \prime}(u)$ and $\chi^{\prime \prime}(u)$ are both positive, which reverses the sign of the integrand in each case. Hence the conclusion. The reverse of the above corollary holds true here.

[Remark. Theorems II and III might have been anticipated intuitionally from the fact that the difference between the variations of the given law and that of Newton is most marked near the center of force. One should therefore expect the apsidal angle to differ from $\pi$ by larger amounts, the nearer the center the orbit extends.]

THEOREM IV. About the center of force there exists a system of concentric spheres, such that those orbits lying wholly between the surfaces of consecutive spheres have their apsidal angles all greater than $\pi$, or else all less than $\pi$.

[The hypothesis upon $P(u)$, viz., $P^{\prime}(u)$ finite save at a finite number of points in any interval, is, however, to be strengthened: $P(u)$ has only a finite number of maxima and minima in any interval.]

By reason of these two hypotheses there exist, in any inter- 
val, only a finite number of values of $r$ for which $P^{\prime}(u)=0$, or $P^{\prime}(u)=\infty$. Select these values as radii of concentric spheres. Then, between consecutive surfaces, $P^{\prime}(u)$ neither vanishes nor becomes infinite, and consequently keeps the same sign. Hence, the difference $\Theta-\pi$ has the same sign for all orbits in the region specified.

\section{Applications of Theorems.}

While all of the above theorems will be utilized in what follows, Theorem I, by reason of its simplicity and generality, offers the most powerful criterion. As will be apparent in the second class of applications to be made, an inspection of the law of force is all that is necessary to ascertain whether the apsidal angle is greater than $\pi$ in some orbits, and less than $\pi$ in others, or whether the difference $\Theta-\pi$ must always have the same sign.

Class I: Motion in the equatorial plane of a spheroid.

Case I. The oblate spheroid. The resultant force varies, not inversely as the square of the distance, but in a much more complicated manner. Denoting by $e$ the eccentricity and by $a$ the major semiaxis of a meridian section, the components of attraction, $X$ and $Y$, parallel to rectangular axes in the equatorial plane are given by *

$$
\begin{aligned}
& \frac{X}{x}=\frac{Y}{y} \\
& =-\frac{3}{2} \frac{k^{2} M \cdot m}{(a e)^{3}}\left[\arcsin \frac{a e}{\sqrt{x^{2}+y^{2}}}-\frac{a e}{\sqrt{x^{2}+y^{2}}} \sqrt{1-\frac{a^{2} e^{2}}{x^{2}+y^{2}}}\right],
\end{aligned}
$$

$m$ being the mass of the attracted particle, $M$ that of the spheroid, and $x$ and $y$ the coordinates of the particle, the origin being at the center of the spheroid. Now (18) shows that the force is central; and, introducing the variable $u$, it is found that

$$
P(u)=\frac{K}{u^{3}}\left[\arcsin a e u-a e u \sqrt{1-(\alpha e u)^{2}}\right],
$$

where $K$ is a positive constant. The right member of (19) is

\footnotetext{
* Moulton, F. R. Introduction to celestial mechanics, pp. 123 ; 118.
} 
representable by a power series in aeu, convergent for all points exterior to the spheroid; thus

$$
P(u)=\sum_{n=0}^{n=\infty} A_{n}(\alpha e u)^{2 n},
$$

where the $A_{n}$ are all positive. Evidently, then, for all exterior points, $P^{\prime}(u)$ is positive and increases with $u$; consequently Theorems I and II apply.

Hence, every orbit described in the equatorial plane of an attracting oblate spheroid has its apsidal angle greater than $\pi$; and, the nearer the center of force the orbit lies, the greater the apsidal angle.

This finds an immediate application in the motion of the fifth satellite of Jupiter, whose orbit deviates exceedingly little from the equatorial plane of the planet. The oblateness of Jupiter is considerable, so that $P(u)$ differs from the newtonian term in the series (20) very markedly for large values of $u$. This, with the fact that the satellite's orbit is very near the planet, should explain the large difference between its apsidal angle and $\pi$, amounting in a year to about $900^{\circ}$.

This "advance of the line of apsides" has also been fully explained from the standpoint of perturbations,* the orbit being regarded as an ellipse whose elements vary ; the difference between the apsidal angle and $\pi$ being considered as a forward rotation of the major axis.

Case II. The prolate spheroid. The attraction upon a particle in the equatorial plane is given by $\dagger$

$$
P(u)=\frac{K}{u^{3}}\left[a e u \sqrt{1+(a e u)^{2}}-\log \left(a e u+\sqrt{1+(a e u)^{2}}\right)\right]
$$

$K$ being a positive constant, and $a$ and $e$ having the same meanings as in Case I. The right member of (21) is developable as a power series in aeu,

where

$$
P(u)=\sum_{n=0}^{n=\infty} B_{n}(\alpha e u)^{2 n},
$$

$$
B_{n}=\frac{1 \cdot 3 \cdot 5 \cdots(2 n-1)}{2 \cdot 4 \cdot 6 \cdots(2 n)} \cdot \frac{(-1)^{n}}{2 n+3} .
$$

* Moulton, loc. cit., pp. 232-233.

† Moulton, loc. cit., p. 123. 
This series is convergent for aeu $<1$; whether this includes all exterior points depends upon the value of $e$.

Since the first coefficient of $P^{\prime}(u)$ is negative, it is evident that $P^{\prime}(u)<0$ for sufficiently small values of $u$. Hence all orbits lying outside of a certain circle have their apsidal angles less than $\pi$.

Again, since the ratio of consecutive coefficients in $P^{\prime \prime}(u)$ is

$$
\frac{(2 n+1)^{2}(2 n+3)}{(2 n-1)(2 n)(2 n+5)}(-1),
$$

which has its greatest numerical value, $\frac{45}{14}$, for $n=1$, it follows that for aeu $<\sqrt{\frac{14}{4}}$, if the terms of $P^{\prime \prime}(u)$ be grouped in pairs, each pair will have a negative sum. So that, for $u$ sufficiently small, $P^{\prime}(u)$ is a decreasing function; therefore the apsidal angle decreases toward the center of force.

Finally, calling aeu $=\lambda$,

$$
P^{\prime}\left(\frac{\lambda}{a e}\right)=\frac{3 K(a e)^{3}}{\lambda^{4} \sqrt{1+\lambda^{2}}}\left[\log \left(\lambda+\sqrt{\lambda^{2}+1}\right)-\left(\lambda+\frac{1}{3} \lambda^{3}\right)\right],
$$

which can be shown to be everywhere negative. Thus, let $\log \left(\lambda+\sqrt{\lambda^{2}+1}\right)-\left(\lambda+\frac{1}{3} \lambda^{3}\right)=\Phi(\lambda)$. Then

$$
\Phi(0)=0, \Phi^{\prime}(\lambda)=\frac{1}{\sqrt{1+\lambda^{2}}}-\left(1+\lambda^{2}\right) .
$$

Since $\Phi^{\prime}(\lambda)<0$ for every real value of $\lambda, \Phi(\lambda)<0$ for $\lambda>0$. Hence, the apsidal angle is less than $\pi$ in all the trajectories.

Class II. Orbits for various simple laws.

The criterion of Theorem I yields especially simple results when applied to the following types of laws of force : If

Case I. Force varies inversely as the $n^{\text {th }}$ power of the distance.

$$
u^{2} P(u)=k^{2} \frac{1}{r^{n}}, \quad P(u)=k^{2} u^{n-2},
$$

and $P^{\prime}(u)=(n-2) k^{2} u^{n-3}$. Three cases arise :

(1) $n=2$; Newton's law, $\Theta=\pi$.

(2) $n>2 ; P^{\prime}(u)>0, P^{\prime \prime}(u) \geqq 0$. Hence, theorems I and II both apply. $\Theta>\pi$, and increases toward the center.

(3) $n<2$; $P^{\prime}(u)<0, P^{\prime \prime}(u)>0$. Theorem I applies, but not Theorem III. $\Theta<\pi$. 
In many of the cases included here it is quite impossible to evaluate in closed form the definite integral defining $\Theta$. Thus, under (2) are included the well known "inverse fifth power," which leads to elliptic integrals; also, all the inverse higher powers leading to abelian and hyperabelian integrals. By Theorem I all these integrals are greater than $\pi$ and increase with the apsidal values of $u$.

Under (3) are included: The "inverse first power," which leads to logarithms, and which is usually omitted from the treatises; the simple law of the "direct first power," where $\Theta=\frac{1}{2} \pi$; the case of the constant force, leading to an elliptic integral, and the higher direct powers with their involved integrals. These apsidal angles are, however, by Theorem I, all less than $\pi$. since

Case II. Force varies as $\log 1 / r^{m}$ and inversely as $r^{n}$. Here, $u^{2} P(u)=\left(k^{2} m / r^{n}\right) \log u, P^{\prime}(u)=k^{2} m\left[(n-2) \log u \cdot u^{n-3}+u^{n-3}\right]$, or

$$
P^{\prime}(u)=k^{2} m u^{n-3}[1+(n-2) \log u] .
$$

From (23) it is evident that for $n=2, P^{\prime}(u)>0$ for $u>0$; for $n>2, P^{\prime}(u) \geqslant 0$ according as $\log u \gtrless-1 /(n-2)$, and for $n<2, P^{\prime}(u) \gtrless 0$, according as $\log u \lessgtr 1 /(2-n)$. That is, if $n>2, \Theta>\pi$ in orbits within a certain sphere; if $n=2, \Theta>\pi$ in all orbits; if $n<2, \Theta>\pi$ in orbits without a certain sphere. The opposite inequalities hold in other parts of space.

Case III. Force varies as $e^{\phi(u)}$ and inversely as $r^{n}$. In this case, since $P^{\prime}(u)=k^{2} u^{n-2}\left[\phi^{\prime}(u)+(n-2) u\right] e^{\phi(u)}$, it follows that if $\phi(u)$ be everywhere real, $P^{\prime}(u)$ has the same sign as $\phi^{\prime}(u)+(n-2) u$. While a discussion of the cases arising when $\phi(u)$ is arbitrary is of small importance, the following special cases may well be noted. If $\phi^{\prime}(u)>0$ for $u>0$, the inequalities of Case II hold; if $\phi^{\prime}(u)$ is everywhere negative, those inequalities are to be reversed.

The laws of force which have been examined, while only a few, embrace most of those commonly considered in treatments of central force problems, and the simplicity of the criterion in these cases encourages the hope that it may be found useful elsewhere.

Williams College, May, 1907. 